\title{
Parent Artery Reconstruction for Large or Giant Cerebral Aneurysms Using the Tubridge Flow Diverter: A Multicenter, Randomized, Controlled Clinical Trial (PARAT)
}

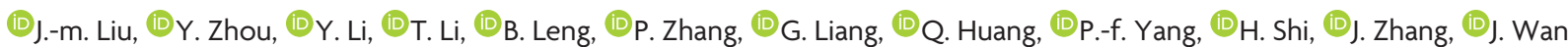

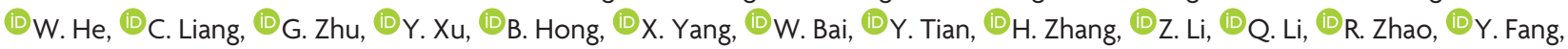 \\ and ${ }^{\circ}$ K. Zhao, for the PARAT investigators \\ i $\mathrm{O}^{\mathrm{E}}=\frac{\mathrm{EBM}}{1}$
}

\begin{abstract}
BACKGROUND AND PURPOSE: Although flow diverters have been reported with favorable clinical and angiographic outcomes in various literatures, randomized trials determining their true effectiveness and safety are still in lack. The Parent Artery Reconstruction for Large or Giant Cerebral Aneurysms Using the Tubridge Flow Diverter (PARAT) trial was designed to evaluate the safety and efficacy of the Tubridge flow diverter in the treatment of large or giant aneurysms in comparison with Enterprise stent-assisted coiling.
\end{abstract}

MATERIALS AND METHODS: This prospective, multicenter, randomized trial was conducted at 12 hospitals throughout China. Enrolled adults with unruptured large/giant intracranial aneurysms were randomly assigned (1:1) to receive either Enterprise stent-assisted coiling or Tubridge flow diverter implantation. The primary end point was complete occlusion at 6-month follow-up, while secondary end points included technical success, mortality, target vessel-related stroke, aneurysm bleeding, in-stent stenosis, parent artery occlusion, and the frequency of all adverse events.

RESULTS: Among 185 enrolled subjects, 41 withdrew before procedure initiation. Overall, 82 subjects underwent Tubridge implantation, and 62 subjects were primarily treated with stent-assisted coiling. The results of 6-month follow-up imaging included complete occlusion rates of $75.34 \%$ versus $24.53 \%$ for the Tubridge and stent-assisted coiling groups, respectively, with a calculated common odds ratio of 9.4 (95\% confidence interval, 4.14-21.38; $P<.001$ ). There was a higher, nonsignificant frequency of complications for Tubridge subjects. Multivariate analysis showed a decreased stroke rate at the primary investigational site, with a marginal $P$ value $(P=.051)$.

CONCLUSIONS: This trial showed an obviously higher rate of large and giant aneurysm obliteration with the Tubridge FD over Enterprise stent-assisted coiling. However, this higher obliteration rate came at the cost of a nonsignificantly higher rate of complications. Investigational site comparisons suggested that a learning curve for flow-diverter implantation should be recognized and factored into trial designs.

ABBREVIATIONS: FD = flow diverter; LTF = lost-to-follow-up; PARAT = Parent Artery Reconstruction for Large or Giant Cerebral Aneurysms Using the Tubridge Flow Diverter; SAC $=$ stent-assisted coiling

arge $(10-25 \mathrm{~mm})$ or giant $(\geq 25 \mathrm{~mm})$ aneurysms are a very challenging subtype among intracranial aneurysms, with

Received January 16, 2018; accepted after revision February 26

From the Department of Neurosurgery, (J.-m.L., Y.Z., P.-f.Y., B.H., Y.X., Q.L., R.Z., Y.F., K.Z., Q.H.), Shanghai Changhai Hospital, Shanghai, China; Department of Neurosurgery (Y.L., X.Y.), Tiantan Hospital, Beijing, China; Department of Interventional Radiology (T.L., W.B.), Henan Provincial People's Hospital, Henan Province, China; Department of Neurosurgery (B.L., Y.T.), Huashan Hospital, Shanghai, China; Department of Neurosurgery (P.Z., H.Z.), Xuanwu Hospital, Beijing, China; Department of Neurosurgery (G.L., Z.L.), General Hospital of Shenyang Military, Shenyang Province, China; Department of Neurosurgery (H.S.), First Clinical Hospital affiliated with Harbin Medical University, Heilongjiang Province, China; Department of Neurosurgery (J.Z.), Second Affiliated Hospital of Zhejiang University, Hangzhou Municipality, Zhejiang, China; Department of Neurosurgery (J.W.), Renji Hospital, Shanghai, China; Department of Neurosurgery (W.H.), Second Affiliated Hospital of Guangzhou Medical University, Guangzhou, China; Department of Neurosurgery (C.L.), First Hospital of China Medical University, Shenyang Municipality, Liaoning, China; and Department of Neurosurgery (G.Z.), Southwest Hospital, Chongqing Municipality, China. much higher risks of rupture and poorer outcomes compared with small aneurysms. ${ }^{1,2}$ Despite recent technical advances, the treatment of large or giant aneurysms remains technically disappointing, with high complication and/or recanalization rates. ${ }^{3-6}$

Jin-min Liu and Yu Zhou contributed equally to this work.

The PARAT investigators are listed in the On-line Appendix.

The trial was funded by the National Research and Development Project of Key Chronic Diseases (Grant No. 2016YFC1300700), the National Science and Technology Supporting Program (2011BA108B14), and the project of the Shanghai Science and Technology Commission (11DZ1921603) and was sponsored by unrestricted grants from MicroPort NeuroTech Co, Shanghai, China.

Please address correspondence to Jian-min Liu, Department of Neurosurgery, Changhai Hospital, Second Military Medical University, 168 Changhai Rd, Shanghai 200433, China; e-mail: liul18@vip.163.com

\footnotetext{
- Indicates open access to non-subscribers at www.ajnr.org

$\equiv$ Indicates article with supplemental on-line appendix and tables.

EBM Evidence-Based Medicine Level 1.

http://dx.doi.org/10.3174/ajnr.A5619
} 
In recent years, various flow diverter (FD) devices, such as the Pipeline Embolization Device (Covidien, Irvine, California), the Silk flow diverter (Balt Extrusion, Montmorency, France), the Flow-Redirection Endoluminal Device (FRED; MicroVention, Tustin, California), and the Surpass stent (Stryker Neurovascular, Kalamazoo, Michigan), have been increasingly used in $>50$ countries. These devices were believed to improve long-term effectiveness, due to their capability to alter intrasaccular hemodynamics. ${ }^{7}$ Unfortunately, reported clinical results have varied significantly, with aneurysm occlusion rates and periprocedural complication rates ranging from $49 \%$ to $93.4 \%$ and $2.8 \%$ to $11 \%$, respectively. On the other hand, there is only 1 randomized controlled trial reported until now (Flow Diversion in Intracranial Aneurysm Treatment [FIAT]), which showed very high complication rates and below-expectation effectiveness. ${ }^{8}$ These results confused neurointerventionalists about the true effectiveness and safety of FDs. The recent publication of Raymond et $\mathrm{al}^{8}$ echoed these sentiments by suggesting that more randomized trials are needed to determine the role of flow diversion in the management of aneurysms.

In 2012, a lack of prospective data and reports of significant adverse outcomes associated with FD use in treating intracranial aneurysms fueled our interest in leading a multicenter, prospective, randomized, controlled trial (Parent Artery Reconstruction for Large or Giant Cerebral Aneurysms Using the Tubridge Flow Diverter [PARAT]) assessing FD treatment of large and giant intracranial aneurysms. In contrast to the FIAT trial, the PARAT trial focused on unruptured large or giant internal carotid artery or vertebral artery aneurysms, the traditional indication for FD implantation. By comparing it with a well-established treatment, stent-assisted coiling (SAC), we attempted to characterize the safety and effectiveness of the Tubridge FD (MicroPort NeuroTech, Shanghai, China) in this specific subset of intracranial aneurysms.

\section{MATERIALS AND METHODS Study Design and Participants}

The PARAT trial used a prospective, multicenter, parallel-group design, with balanced randomization (1:1). The primary trial purpose was to compare outcomes of subjects with unruptured large/ giant intracranial aneurysms who were treated with either Enterprise SAC (Codman \& Shurtleff, Raynham, Massachusetts) or Tubridge FD implantation. The study was conducted in 12 centers throughout China, each of which was required to have performed $>30$ stent-assisted coiling procedures per year during the previous 5 years. The PARAT trial protocol was approved by all relevant local ethics boards. ${ }^{9}$ Site investigators generated trial data, with monitoring and data base maintenance completed by a commercial clinical research organization. The corresponding author had full access to all trial data and had final authority for key decisions relevant to this publication.

At each trial center, prospective subjects were screened for trial eligibility, based on having an unruptured ICA or vertebral artery saccular aneurysm (including recanalized aneurysms) measuring $\geq 10 \mathrm{~mm}$ in maximum diameter and $\geq 4 \mathrm{~mm}$ across the aneurysm neck. Investigators recruited only those who met all inclusion criteria and none with the exclusion criteria. Patients with rup- tured aneurysms or other intracranial diseases were excluded. Detailed inclusion and exclusion criteria are listed in On-line Table 1. Investigational sites did not record or retain a log of patients who were screened for eligibility. Written informed consent was obtained from each participant before enrollment.

\section{Randomization and Masking}

After we obtained each prospective subject's informed consent, treatment allocation was initiated by a researcher contacting a clinical research associate who was independent of the patientrecruitment procedure. Subject randomization was accomplished through an interactive Web response system, which was developed by information technology managers from an independent clinical research organization under the instruction of a clinical trial statistician. The randomization scheme included built-in stratification by the participating center, aneurysm size ( $\leq 15 \mathrm{~mm}$ versus $>15 \mathrm{~mm}$ ), and aneurysm location (anterior circulation versus posterior circulation). The on-line central randomization ensured that the allocation sequence was concealed from investigators who were recruiting patients before the decision to randomize. Treatment groups were balanced using minimization criteria.

Masking of the randomization results to local investigators or trial participants was impossible to achieve. However, members of an independent Core Laboratory and those who followed up with trial subjects via telephone or face-to-face on-site interviews were blinded as to treatment allocation.

\section{Interventions, Treatment, and Evaluation Procedures}

For all recruited patients, dual-antiplatelet drugs (300 mg/day of aspirin plus $75 \mathrm{mg}$ /day of clopidogrel) were prescribed for at least 3 days before the pivotal procedure. All FD placement procedures were performed with the patient under general anesthesia and via a transfemoral approach. After sheath placement, heparin (100 $\mathrm{u} / \mathrm{kg}$ ) was administered to maintain an activated clotting time of 250-350 seconds throughout the procedure. Next, a suitable guiding catheter (7F for subjects to be treated with Tubridge and coils) was placed in the distal internal carotid or vertebral artery. Subsequently, treatment group subjects underwent Tubridge FD implantation alone or in combination with bare coils, while control group subjects were treated by Enterprise stent-assisted coiling with bare coils.

The Tubridge Flow Diverter, developed by MicroPort NeuroTech, Shanghai, China, is designed to encourage the formation of intra-aneurysmal clot, while concurrently repairing and reconstructing the parent artery. Previously, we have characterized the Tubridge FD and its structural differences from the Pipeline and Silk flow diverters, such as the platinum-iridium material used for the radiopaque microfilaments, more braided microfilaments for the large-sized FD, and a decreased incidence of shortening (Table 1). ${ }^{9}$ Deployment of the Tubridge FD requires a combination "push" and "pull" technique to ensure full opening of the device and to increase the metal coverage across the aneurysm neck, as previously described. ${ }^{9}$

The approach and timing of coil placement and the number of Tubridge devices implanted were left to the discretion of each operator. For control subject aneurysms, Enterprise stents were 
Table 1: Structural comparison between different FDs

\begin{tabular}{|c|c|c|c|c|c|c|c|}
\hline \multirow[b]{2}{*}{ FD Type } & \multirow[b]{2}{*}{ Size } & \multicolumn{2}{|c|}{ Braided Microfilaments $^{a}$} & \multirow{2}{*}{$\begin{array}{l}\text { Radiopaque } \\
\text { Microfilaments }\end{array}$} & \multirow{2}{*}{$\begin{array}{l}\text { Flared } \\
\text { End }\end{array}$} & \multirow{2}{*}{$\begin{array}{l}\text { Metal } \\
\text { Coverage }\end{array}$} & \multirow[b]{2}{*}{ Retrievable } \\
\hline & & No. & Material & & & & \\
\hline Pipeline & $3-5.5 \mathrm{~mm}$ & 48 & $\begin{array}{l}75 \% \text { cobalt chromium } \\
\text { and } 25 \% \text { platinum }\end{array}$ & NA & No & $30 \%-35 \%$ & Yes $^{\mathrm{b}}$ \\
\hline Silk & $2.5-5 \mathrm{~mm}$ & 48 & Nickel-titanium alloy & 4 Platinum wires & Yes & $35 \%-55 \%$ & Yes \\
\hline Surpass & $2.5-5 \mathrm{~mm}$ & $\begin{array}{l}2.5 \mathrm{~mm}(36) \\
3 \text { and } 4 \mathrm{~mm}(60) \\
5 \mathrm{~mm}(84)\end{array}$ & Cobalt-chromium & 12 Platinum wires & No & $30 \%$ & NA \\
\hline FRED & $2.5-5.5 \mathrm{~mm}$ & $\begin{array}{l}\text { Inner layer (48) } \\
\text { Outer layer (16) }\end{array}$ & Nickel-titanium alloy & 2 Tantalum wires & Yes & NA & Yes \\
\hline Tubridge & $2.5-6.5 \mathrm{~mm}$ & $\begin{array}{l}<3.5 \mathrm{~mm}(46) \\
\geq 3.5 \mathrm{~mm}(62)\end{array}$ & Nickel-titanium alloy & $\begin{array}{l}2 \text { Platinum-iridium } \\
\text { wires }\end{array}$ & Yes & $30 \%-35 \%$ & Yes \\
\hline
\end{tabular}

Note:-NA indicates not applicable.

a Braided microfilaments in this table mean those main wires excluding microfilaments especially for radiopaque usage.

b Pipeline Flex embolization devices are retrievable.

implanted according to the instructions for use of the product, and all aneurysms were embolized (coiled) to achieve maximum packing density. All subject treatments were well-documented, and details included aneurysm shape and dimensions, width of the aneurysmal neck, diameter of the parent artery, and all relevant treatment outcomes. The postoperative antiplatelet regimen was as follows: $<6$ weeks: $300 \mathrm{mg}$ of aspirin $+75 \mathrm{mg}$ of clopidogrel; 6 weeks to 3 months: $100 \mathrm{mg}$ of aspirin $+75 \mathrm{mg}$ of clopidogrel; and, $\geq 3$ months: $100 \mathrm{mg}$ of aspirin indefinitely.

Digital copies of angiograms, including 3D rotational angiography as well as working projection images and other anteroposterior/lateral angiographic images, were collected by the clinical research organization-assigned clinical research associate of each site. These were sent to an independent Core Laboratory for analyses by up to 3 experienced neurointerventionalists ("analysts"). On the first pass, 2 analysts separately reviewed each subject's imaging. If the initial 2 interpretations conflicted, a third analyst provided a tie-breaking assessment. Follow-up angiographic results were classified into 4 categories, according to the immediate degree of embolization: 1) occluded, defined as no contrast filling into the aneurysm sac; 2) improved, defined as decreased contrast filling into the aneurysm sac; 3 ) stable, defined as unchanged contrast filling into the aneurysm sac; and, 4) recanalized, defined as increased contrast filling into the aneurysm sac. Analysts also documented instances of parent artery occlusion or in-stent stenosis.

At 1, 3, and 6 months postimplantation, experienced site investigators followed up with each subject by telephone or by inperson clinical interviews. To provide some level of objectivity, these interviewers were blinded to the treatment allocation. If any adverse events were identified, investigators documented, in full detail, the following information: symptoms, event duration and severity, possible causes and associations, actions taken, and event resolution/final outcomes. A fully independent Clinical Events Committee evaluated all investigator-documented adverse events and categorized them according to the most likely causal relationship: procedure-related, device-related, disease-related, or unrelated to either disease, device, or procedure.

\section{Study Outcomes}

The primary end point was complete aneurysm occlusion at the 6-month follow-up. During reviews of 3D rotational angiography and other angiographic images, members of the Core Laboratory consistently followed precise analytic criteria for establishing the final effectiveness end point. Only those aneurysms exhibiting no contrast filling in the aneurysm were judged as meeting the complete occlusion designation. End point analysis was expressed as percentage occurrence within the test and control groups.

Secondary end points included the following:

1) Immediate technical success rate, which included successful device delivery, exact stent positioning, and full expansion of the devices.

2) Death or stroke related to target vessel (assessment time points: 30 days, 90 days, and 1 year postoperation). Stroke was defined as sudden symptoms and signs of a focal cerebral function deficit associated with cerebral circulation disorders and including hemorrhagic or ischemic stroke. Hemorrhagic stroke was defined as acute extravasation of blood into the brain parenchyma or subarachnoid space with associated neurologic symptoms, whereas ischemic stroke was defined as rapid onset of a new focal neurologic deficit or rapid worsening of an existing focal neurologic deficit with clinical evidence of infarction not attributable to a nonischemic etiology (not associated with brain infection, trauma, tumor, seizure, severe metabolic disease, or degenerative neurologic disease).

3) Aneurysm bleeding rate (assessment time points: 30 days, 90 days, and 1 year postoperation), including intraoperative rupture and delayed aneurysm rupture confirmed by CT.

4) The rate of in-stent stenosis (assessment time point: 6 months postoperation).

5) The rate of parent artery occlusion (assessment time point: 6 months postoperation).

6) General adverse events (assessment time points: 30 days, 90 days, and 1 year postoperation).

\section{Statistical Analysis}

On the basis of an assumption of a 20\% lost-to-follow-up (LTF) rate, a 124-subject sample size was planned (62 per group) to obtain a valid result (2-tailed test, significance level of $\alpha=.05$, power of $1-\beta=0.80)$. However, because postrandomization subject withdrawals were higher than anticipated, the independent Data Monitoring Committee of the trial suggested a larger sample size to reach the required minimum number for each group (62 subjects). Differences between the PARAT trial protocol and a final report were included in the statistical analysis plan.

AJNR Am J Neuroradiol 39:807-16 May 2018 www.ajnr.org 
Data analyses included a modified intention-to-treat approach based on a population of subjects who were recruited and treated (full analysis set). Those who left the trial immediately after randomization and who did not undergo the pivotal procedure were removed from this analysis. Those who completed treatment and the entire follow-up protocol constituted the perprotocol set, while those who were treated and had at least 1 safety evaluation were included in the safety set. To verify intergroup balance, the statisticians compared the baseline characteristics of subjects between the 2 study arms. Proportions were used for categoric variables, and medians with interquartile ranges were used for continuous variables. The categoric variables were compared between study arms using the $\chi^{2}$ or Fisher exact test. For continuous variables, the $t$ test or Mann-Whitney rank test, as applicable, was used according to the distribution of the data.

Analysis of the primary effectiveness end point involved a comparison of complete occlusion rates at the 6-month follow-up between the treatment and control groups in the full analysis set using the $\chi^{2}$ method in combination with multivariable logistic regression adjusted for subject age (60 years or younger versus older than 60 years) and aneurysm size ( $\leq 15$ versus $>15 \mathrm{~mm}$ ). A sensitivity analysis was performed to assess the effect of missing data (ie, those who were partially or wholly LTF after undergoing the pivotal procedure). A per-protocol set analysis was also performed excluding the following: subjects who were enrolled in the trial but who did not undergo the index procedure, those in whom the procedure failed, and those who did not reach the primary end point because they were deemed LTF. Secondary outcomes were compared between groups in the safety set using the $\chi^{2}$ or Fisher exact test, as applicable. An additional multivariable logistic regression was performed to explore factors potentially affecting subject outcomes.

Statistical analyses were conducted by statisticians at the Institute of Clinical Evaluation, affiliated with Beijing University, and the data were analyzed using Statistical Analysis System software, Version 9.2 (SAS Institute, Cary, North Carolina). All tests were 2-sided, and a $P$ value $<.05$ was considered statistically significant.

An independent Data and Safety Monitoring Committee had unrestricted access to trial data, to allow periodic monitoring of trial progress. This trial was registered on the Chinese Clinical Trial Registry: ChiCTR-TRC-13003127.

\section{RESULTS}

\section{Subject Characteristics and Disposition}

Between December 18, 2012, and May 9, 2014, one hundred eighty-five subjects were enrolled and randomized. The distribution of subjects among the investigational sites is shown in On-line Table 2. Of considerable surprise and prompting a midstudy revision of enrollment strategy was the high proportion of subjects $(n=41)$ exiting the study after randomization. Details of these LTF subjects are found in the final part of this article in a discussion of trial limitations as well as in the Figure.

Pivotal treatments were initiated in the remaining 144 subjects who formed the full analysis set based on a principle of modified intention-to-treat. Due to a tortuous parent artery or a wide an- eurysm neck, investigators failed to catheterize the parent artery or deliver devices across the aneurysm neck in 6 instances ( 3 per trial group). Of 138 treated subjects, 5 died; 1 withdrew after serious procedure-related complications; and 6 were defined as LTF without reason. The remaining 126 subjects were included in the per-protocol analysis. There were no subject crossovers among trial groups. Treatment and follow-up details are shown in On-line Table 3.

A comparison of baseline characteristics between the 2 groups in the full analysis set showed similar distributions except for subject age $(P=.036$, Table 2$)$, which was not considered a prognostic factor for the primary outcome. Because a high proportion of participants quit after randomization, we also compared baseline characteristics between the 41 excluded subjects and the 144 subjects in the full analysis set and determined that the distributions were comparable (On-line Table 4).

\section{Primary Outcome}

As shown in Table 3, in the 6-month angiographic follow-up, aneurysms treated with Tubridge FDs were associated with a favorable shift toward a complete occlusion rate. In the per-protocol analysis of 126 cases for the primary effectiveness end point, the complete occlusion rate was $75.34 \%$ (55/73) for the Tubridge group and $24.53 \%$ (13/53) for the Enterprise control group, with an adjusted common odds ratio of 9.31 (95\% confidence interval, $4.00-21.66 ; P<.001$ ). Unadjusted results and sensitivity analyses also showed superior results for the Tubridge group. Table 4 lists 6-month $100 \%$ aneurysm occlusion rates in the context of aneurysm location and size.

\section{Secondary Outcomes}

Immediate technical success rates did not differ significantly between the 2 trial groups. Three procedures in each group failed due to difficulties in advancing a microcatheter into the distal arteries. The calculated technical success rates were 96.34\% (79/82) and 95.16\% (59/62) in the Tubridge FD group and Enterprise control group, respectively, with a common odds ratio of 1.34 (95\% CI, $0.26-6.87 ; P=.726$ ).

Trial statisticians calculated a trend toward an increased but nonsignificant risk of complications for subjects in the Tubridge group. Hemorrhagic stroke occurred in 7 subjects, including 5/82 (6.1\%) in the Tubridge group and 2/62 (3.23\%) in the control group. Ischemic stroke, related to target vessels, occurred in $8 / 82(9.76 \%)$ of the Tubridge group and $6 / 62$ (9.68\%) of the control group. These complications resulted in 4 deaths: Three were Tubridge subjects and 1 was an Enterprise-treated subject. In addition, 1 subject in each of the 2 trial groups died during follow-up for reasons unrelated to target vessels. Overall, the rates of death or stroke related to target vessels in 1-year follow up were $14.52 \%$ and $17.07 \%$ in the control and Tubridge groups, respectively. Statistically, there was no significant difference between the groups $(P=.678)$.

The aneurysm bleeding rate was comparable between groups, with $3.66 \%$ versus $1.61 \%$ for the Tubridge group and Enterprise control group, respectively $(P=.634)$. Overall, general adverse events occurred in $46 / 82(56.10 \%)$ of the Tubridge 


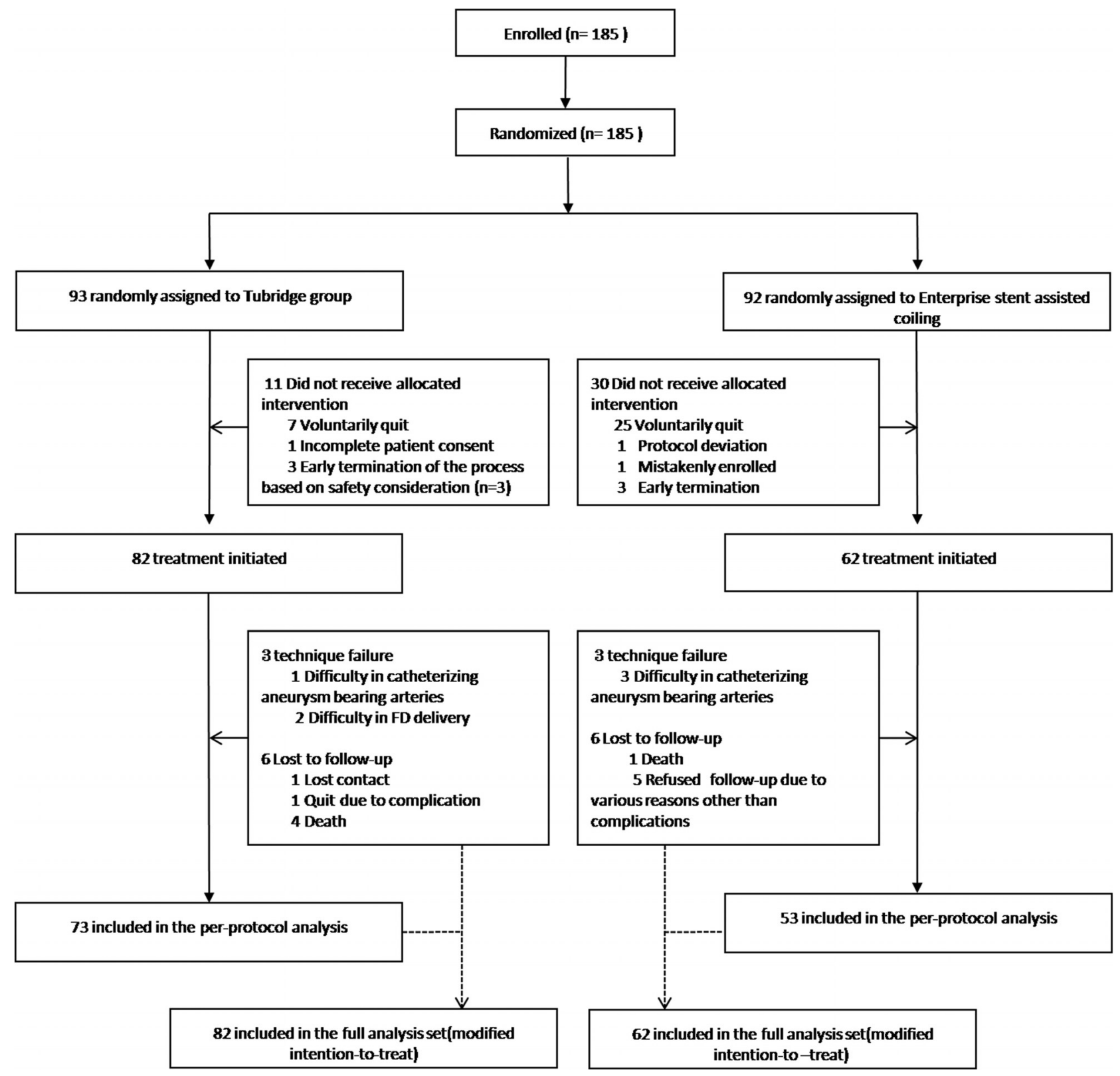

FIGURE. Flow diagram of the PARAT trial.

group and 33/62 (53.23\%) of the Enterprise control group; among these, most were symptoms unrelated to the device, index procedure, or aneurysmal disease, such as headache, vomiting, or fever and there was no significant difference between the groups at the 30-day, 90-day, or 1-year follow-up. There was a trend toward a higher rate of in-stent stenosis or parent artery occlusion in the Tubridge group, but the difference was not statistically significant (Table 5).

\section{Additional Complication Details}

As shown in On-line Table 5, target vessel-related stroke, both ischemic and hemorrhagic, occurred in 21 subjects. Eight occurred during the procedure; 9 , within the first 30 days after the index procedure (procedure-related); and the remaining 4 cases, at 1,2,3, and 7 months posttreatment.

Intraoperative bleeding occurred in 3 subjects. One Tubridge subject had a microwire injury to a distal artery and died, while the other 2 subjects with intraoperative bleeding (1 per group/mRS of 1 at follow-up) recovered well after prompt coil deployment immediately after the onset of rupture. The Clinical Events Committee concluded that these complications, though unrelated to the Tubridge and Enterprise devices per se, were classified as procedure-related complications.

Periprocedural bleeding occurred in 4 cases ( 3 Tubridge/ 1 Enterprise). Delayed aneurysm rupture accounted for 2 complications (both large or giant aneurysms treated with FD alone). Of the remaining 2 cases, 1 Enterprise subject presented with cerebral hematoma and 1 Tubridge subject presented with mild subarachnoid hemorrhage in the Sylvian fissure. Both were thought to be unrelated to aneurysm rupture. The patient with a cerebral hematoma died due to prehospital delay, while the other one recovered well.

Overall, there were 14 cases of target vessel-related ischemic

AJNR Am J Neuroradiol 39:807-16 May 2018 www.ajnr.org

811 
stroke ( 8 Tubridge/6 Enterprise). Five subjects experienced intraoperative ischemia; another 5, within the first 30 days; and the remaining 4 , at 1-7 months posttreatment.

Among those with intraoperative ischemic events, 3 were Tubridge subjects. One had a stroke after anterior choroidal artery occlusion due to coil introduction; another had a frontoparietal acute infarction due to parent artery occlusion caused by intrastent thrombosis; and, the other one was thought to be the result of a thromboembolic event. All of them resulted in contralateral limb weakness, and symptoms resolved in 1 case. In the 2 control subjects, patients had ischemic symptoms after uneventful procedures. Thromboembolic events were considered after further CT and angiographies, and both recovered well after medical therapy.

Among 5 subjects with periprocedural thromboembolic events (2 Tubridge/3 Enterprise), ischemic symptoms were all identified within 1 week after the procedure. Symptoms resulting from these periprocedural ischemia events were characterized as mild and transient, with all events resolving well after aggressive antiplatelet therapy.

There were no hemorrhagic events after the periprocedural period. However, delayed ischemic stroke occurred in 4, including 3 Tubridge subjects and 1 Enterprise subject. For the $3 \mathrm{Tu}-$ bridge subjects, 2 strokes were identified with parent artery occlusion after further examination and the patients were treated conservatively; and the other 2 patients ( 1 per group) had ischemic symptoms 1 and 2 months postprocedure, with patent parent arteries. All these symptoms improved during the follow-up period.

Overall, among all these complications, there were 4 deaths and 2 disabilities identified during the last follow-up. The procedure-related mortality rate was $3.66 \%(3 / 82)$ in the Tubridge group versus $1.61 \%(1 / 62)$ in the Enterprise group, while the procedure-related morbidity rate was $2.4 \%(2 / 82)$ in the Tubridge group versus zero in the control group in 1-year follow-up.
Table 2: Baseline characteristics of the full analysis set ${ }^{\mathrm{a}}$

\begin{tabular}{|c|c|c|}
\hline Characteristics & $\begin{array}{c}\text { Tubridge Group } \\
n=82\end{array}$ & $\begin{array}{c}\text { Enterprise Group } \\
\qquad n=62\end{array}$ \\
\hline Mean age $(y r)$ & $52.11(10.31)$ & $55.66(9.53)$ \\
\hline Sex ratio (male/female) & $21: 61$ & 13:49 \\
\hline \multicolumn{3}{|l|}{ Medical history } \\
\hline Cerebrovascular stroke & $9(10.98 \%)$ & $8(12.90 \%)$ \\
\hline Coronary artery disease & $1(1.22 \%)$ & $4(6.45 \%)$ \\
\hline Hypertension & $41(50.0 \%)$ & $25(40.32 \%)$ \\
\hline Hypercholesterolemia & $4(4.88 \%)$ & $3(4.84 \%)$ \\
\hline Diabetes & $5(6.10 \%)$ & $2(3.23 \%)$ \\
\hline Current/previous smoking & $12(14.63 \%)$ & $7(11.29 \%)$ \\
\hline Systolic blood pressure (median) (mm Hg) & $129.50(120.0-143.0)$ & $129.50(120.0-138.0)$ \\
\hline \multicolumn{3}{|l|}{ Aneurysm location ${ }^{b}$} \\
\hline Anterior circulation & $77(96.25 \%)$ & $59(98.33 \%)$ \\
\hline Posterior circulation & $3(3.75 \%)$ & $1(1.67 \%)$ \\
\hline Aneurysm size (median) & $18.00(13.14-26.0)$ & $17.14(12.44-24.74)$ \\
\hline \multicolumn{3}{|l|}{ Aneurysm size classification } \\
\hline Large (10-15 mm) & $30(36.59 \%)$ & $24(38.71 \%)$ \\
\hline Very large or giant ( $>15 \mathrm{~mm})$ & $52(63.41 \%)$ & $38(61.29 \%)$ \\
\hline
\end{tabular}

${ }^{a}$ Data are No. (\%), median (interquartile range), or mean (SD).

${ }^{\mathrm{b}}$ Of the 6 patients with failed procedures, locations of 2 aneurysms were absent.

\section{Multivariate Analysis}

To further explore the factors affecting angiographic outcomes and complication occurrences, trial statisticians performed multivariate analysis, including subject age, aneurysm size, aneurysm location (anterior circulation versus posterior circulation), and treatment technique (FD alone; FD + coils; and Enterprise stent-assisted coiling). The primary investigational site (Shanghai Changhai hospital) showed a decreased stroke rate compared with other trial centers, with a marginal $P$ value $(\mathrm{OR}=$ 4.81; 95\% CI, 0.991-23.335; $P=.051$ ).

\section{DISCUSSION}

Since the introduction of flow diverters to the global neurovascular community,

Table 3: Sensitivity analyses on the composite primary outcome

\begin{tabular}{|c|c|c|c|c|c|c|c|c|}
\hline \multirow[b]{2}{*}{ Factoring in Subjects Who Missed F/U } & \multicolumn{2}{|c|}{ 6-Mo Occlusion Rate } & \multicolumn{3}{|c|}{ Unadjusted Result } & \multicolumn{3}{|c|}{ Adjusted Result ${ }^{a}$} \\
\hline & $\begin{array}{l}\text { Tubridge } \\
\text { Group }\end{array}$ & $\begin{array}{l}\text { Control } \\
\text { Group }\end{array}$ & OR & $95 \% \mathrm{Cl}$ & $\begin{array}{c}P \\
\text { Value }\end{array}$ & OR & $95 \% \mathrm{Cl}$ & $\begin{array}{c}P \\
\text { Value }\end{array}$ \\
\hline Missing F/U excluded (PPS) & $55 / 73,75.34 \%$ & $13 / 53,24.53 \%$ & 9.40 & $4.14-21.38$ & $<.001$ & 9.31 & $4.00-21.66$ & $<.001$ \\
\hline Missing F/U counted as complete occlusion & $64 / 82,78.05 \%$ & $22 / 62,35.48 \%$ & 6.47 & $3.09-13.52$ & $<.001$ & 6.14 & $2.92-12.91$ & $<.001$ \\
\hline Missing F/U counted as not completely occluded & $55 / 82,67.07 \%$ & $13 / 62,20.97 \%$ & 7.68 & $3.57-16.51$ & $<.001$ & 7.34 & $3.36-16.05$ & $<.001$ \\
\hline $\begin{array}{l}\text { Missing F/U counted as complete occlusion for } \\
\text { control and incomplete occlusion for Tubridge }\end{array}$ & $55 / 82,67.07 \%$ & $22 / 62,35.48 \%$ & 3.70 & $1.85-7.42$ & $<.001$ & 3.49 & $1.73-7.03$ & $<.001$ \\
\hline
\end{tabular}

Note:-F/U indicates follow-up; PPS, per-protocol set.

${ }^{a}$ Adjusted for each subject's age and aneurysm size ( $\leq 15$ versus $>15 \mathrm{~mm}$ ).

Table 4: Data correlating aneurysm location and size with aneurysm complete occlusion rate

\begin{tabular}{lccccc} 
& & & & & \multicolumn{1}{c}{$\begin{array}{c}100 \% \text { Occlusion } \\
\text { Rate at 6-Mo F/U }\end{array}$} \\
\cline { 3 - 6 } \multicolumn{1}{c}{ Aneurysm Location } & No. & Aneurysm Size (mm) & $\begin{array}{c}\text { Proportion of } \\
\text { Giant Aneurysms }\end{array}$ & $\begin{array}{c}\text { Tubridge } \\
\text { Controls }\end{array}$ \\
\hline ICA communicating & 19 & $18.0 \pm 7.6(10.0-33.9)$ & $4 / 19$ & $40.9 \%, 10 / 13$ & $40 \%, 2 / 5$ \\
ICA ophthalmic & 39 & $15.8 \pm 5.6(10.0-30.0)$ & $4 / 39$ & $83.3 \%, 15 / 18$ & $33.3 \%, 6 / 18$ \\
ICA paraclinoid or cavernous & 73 & $21.8 \pm 7.5(10.0-45)$ & $25 / 73$ & $75.7 \%, 28 / 37$ & $13.8 \%, 4 / 29$ \\
ICA petrous & 3 & $21.3 \pm 9.3(15.0-32.0)$ & $1 / 3$ & $50 \%, 1 / 2$ & 0 \\
Vertebral artery & 4 & $14.3 \pm 2.2(12.0-16.3)$ & $0 / 4$ & $33.3 \%, 1 / 3$ & $100 \%, 1 / 1$ \\
\hline
\end{tabular}

Note:-F/U indicates follow-up. 


\begin{tabular}{|c|c|c|c|c|}
\hline Secondary Outcome & $\begin{array}{l}\text { Enterprise } \\
\qquad n=62\end{array}$ & $\begin{array}{c}\text { Tubridge } \\
n=82\end{array}$ & Odds Ratio (95\% Cl) & $P$ Value \\
\hline \multicolumn{4}{|l|}{ Death or target vessel-related stroke } & .726 \\
\hline 30 days & $7 / 62(11.29 \%)$ & $11 / 82(13.41 \%)$ & $0.821(0.299-2.258)$ & .703 \\
\hline 90 days & $8 / 62(12.90 \%)$ & $12 / 82(14.63 \%)$ & $0.864(0.330-2.263)$ & .766 \\
\hline $1 \mathrm{yr}$ & $9 / 62(14.52 \%)$ & $14 / 82(17.07 \%)$ & $0.825(0.332-2.051)$ & .678 \\
\hline \multicolumn{5}{|l|}{ Mortality rate } \\
\hline 30 days & $1 / 62(1.61 \%)$ & $3 / 82(3.66 \%)$ & $0.432(0.044-4.253)$ & .634 \\
\hline 90 days & $1 / 62(1.61 \%)$ & $4 / 82(4.88 \%)$ & $0.320(0.035-2.934)$ & .391 \\
\hline $1 \mathrm{yr}^{\mathrm{a}}$ & $2 / 62(3.23 \%)$ & $4 / 82(4.88 \%)$ & $0.650(0.115-3.668)$ & .699 \\
\hline \multicolumn{5}{|c|}{ Hemorrhagic stroke related to target vessel } \\
\hline 30 days & $2 / 62(3.23 \%)$ & $5 / 82(6.1 \%)$ & $0.531(0.096-2.738)$ & 699 \\
\hline 90 days & $2 / 62(3.23 \%)$ & $5 / 82(6.1 \%)$ & $0.531(0.096-2.738)$ & 699 \\
\hline $1 \mathrm{yr}$ & $2 / 62(3.23 \%)$ & $5 / 82(6.1 \%)$ & $0.531(0.096-2.738)$ & 699 \\
\hline \multicolumn{5}{|l|}{ Ischemic stroke related to target vessel } \\
\hline 30 days & $4 / 62(6.54 \%)$ & $6 / 82(7.32 \%)$ & $0.874(0.236-3.239)$ & .874 \\
\hline 90 days & $5 / 62(8.06 \%)$ & $6 / 82(7.32 \%)$ & $1.111(0.323-3.822)$ & .867 \\
\hline $1 \mathrm{yr}$ & $6 / 62(9.68 \%)$ & $8 / 82(9.76 \%)$ & $0.991(0.325-3.109)$ & .987 \\
\hline \multicolumn{5}{|l|}{ Aneurysm rupture } \\
\hline 30 days & $1 / 62(1.61 \%)$ & $3 / 82(3.66 \%)$ & $0.357(0.072-1.783)$ & .634 \\
\hline 90 days & $1 / 62(1.61 \%)$ & $3 / 82(3.66 \%)$ & $0.357(0.072-1.783)$ & .634 \\
\hline $1 \mathrm{yr}$ & $1 / 62(1.61 \%)$ & $3 / 82(3.66 \%)$ & $0.357(0.072-1.783)$ & .634 \\
\hline \multicolumn{5}{|l|}{ General adverse events } \\
\hline 30 days & $26 / 62(41.94 \%)$ & $42 / 82(51.22 \%)$ & $0.808(0.293-2.229)$ & .269 \\
\hline 90 days & $26 / 62(41.94 \%)$ & $42 / 82(51.22 \%)$ & $0.808(0.293-2.229)$ & .269 \\
\hline 1 year & $33 / 62(53.23 \%)$ & $46 / 82(56.10 \%)$ & $0.938(0.644-1.365)$ & .88 \\
\hline Rate of intrastent stenosis & $2 / 53(3.77 \%)$ & $4 / 73(5.48 \%)$ & $0.676(0.119-3.837)$ & 1 \\
\hline Rate of intrastent thrombosis & $1 / 59(1.69 \%)$ & $6 / 79(7.59 \%)$ & $0.215(0.025-1.839)$ & .273 \\
\hline
\end{tabular}

${ }^{a}$ Two subjects died during follow-up for reasons unrelated to the target vessels (1 each in Tubridge and control groups).

many clinical studies were conducted to evaluate the safety and effectiveness of FDs, such as the Pipeline for Uncoilable or Failed Aneurysms (PUFS), Aneurysm Study of Pipeline in an Observational Registry (ASPIRe), and PITA trials. ${ }^{8,10-18}$ Additional effort to evaluate the effectiveness and safety of FDs versus conventional treatments included the Multicenter Randomized Trial on Selective Endovascular Aneurysm Occlusion with Coils versus Parent Vessel Reconstruction using the SILK Flow Diverter (MARCO POLO), FIAT, Flow Diverter Stent for Endovascular Treatment of Unruptured Saccular Wide-necked Intracranial Aneurysms (EVIDENCE), and Complete Occlusion of Coilable Aneurysms (COCOA) trials. ${ }^{19-22}$ However, the number of studies that focused on FD treatment of large or giant intracranial aneurysms is low. The purpose of the PARAT trial was to compare the safety and effectiveness outcomes in the treatment of ICA or vertebral artery large or giant aneurysms with the Tubridge FD (with and without coiling) versus a more conventional approach using Enterprise SAC. The PARAT trial results suggested that in subjects with ICA or vertebral artery large or giant aneurysms, Tubridge FD implantation had a significantly higher 6-month complete occlusion rate compared with conventional stent-assisted coiling. However, there was a trend toward increased risk of stroke with FD implantation.

The treatment of large or giant intracranial aneurysms has evolved significantly during the past few years. Before the emergence of FDs, parent artery occlusion, coiling alone, and stentassisted coiling were the major treatment modalities for intracranial large or giant aneurysms. According to a meta-analysis by Turfe et $\mathrm{al}^{, 23}$ parent artery occlusion can result in a complete occlusion rate of $93.0 \%$ (95\% CI, 86.0\%-97.0\%). However, par- ent artery occlusion may be a viable treatment option only when there is sufficient compensating blood flow. Even when a balloon occlusion test finding is negative, a $4 \%-15 \%$ complication rate is possible. ${ }^{23}$ In addition, there have been concerns about de novo aneurysm occurrence after carotid occlusion. ${ }^{24}$ In a report by Arambepola et $\mathrm{al}^{25} 4.3 \%$ of patients developed de novo aneurysms within a mean of 9 years. Bypass surgery may reduce the incidence of ischemic events, but the procedure may be too complicated, leading to morbidity and mortality rates as high as $7 \%$ and $13 \%$, respectively. ${ }^{26-28}$ In this trial, parent artery occlusion was not selected as a feasible control treatment because parent artery sacrifice is considered, throughout most hospitals in China, a last and somewhat futile option for treating intracranial aneurysms. Thus, only those cases posing considerable difficulty or possible failure in parent artery reconstruction would be treated with this method as a salvaging effort. Other than with simple and uncomplicated cases, coiling of large or giant aneurysms without a stent is undertaken far less frequently because of anticipated high recanalization rates. ${ }^{23}$

In many prospective multicenter studies, complete occlusion rates at final follow-up varied from $49 \%$ to $93.4 \%$, and 6-month complete occlusion rates ranged from $55.7 \%$ to $93.3 \%$ (On-line Table 6). In the treatment of large or giant aneurysms with FDs, Becske et $\mathrm{al}^{18}$ reported a complete occlusion rate of $76.4 \%$ at 180 days. Chalouhi et $\mathrm{al}^{29}$ reported a complete occlusion rate of $86 \%$ at a median angiographic follow-up of 7 months. On the basis of a meta-analysis of 29 studies, including 1451 patients with 1654 aneurysms, Brinjikji et $\mathrm{al}^{30}$ reported that the complete occlusion rate after FD implantation was 74\% (95\% CI, 63\%-83\%) for large aneurysms and 76\% (95\% CI, 53\%-90.0\%) for giant aneurysms. 
Generally, the complete occlusion rate of $75.3 \%$ in the Tubridge group at 6 months seems comparable with rates reported in previous publications. The complete occlusion rate of $24.5 \%$ for the control group is lower than that reported in previously published articles. In 2 relatively large studies involving large or giant aneurysms, complete occlusion rates of $31 \%$ and $41 \%$ were reported by Sluzewski et $\mathrm{al}^{5}$ and Chalouhi et $\mathrm{al}^{29}$ respectively. The discrepancies between the latter reports and other published results, as noted above, may be related to variations in patient-selection criteria, study methods, trial design, aneurysm characteristics, and evaluation specifications and procedures. Nevertheless, the effectiveness of Tubridge FD treatment appears to be superior to that of conventional stent-assisted coiling and comparable with reported effectiveness outcomes for other FDs.

Other concerns after FD implantation include technical success rates and overall safety, as well as how FD treatment compares with conventional treatment options. The Enterprise stent is thought to be a safe device for aneurysm treatment, and hemorrhagic complications are assumed to be uncommon. ${ }^{31}$ Although exact causes are not well-understood, delayed aneurysm rupture or intraparenchymal hemorrhage after FD implantation has been recognized.

As shown in On-line Table 6, the hemorrhagic stroke and aneurysm rupture rates were as high as $6.9 \%$ and $5.2 \%$, respectively, as reported for multicenter prospective studies. In the International retrospective study of the Pipeline embolization device (IntrePED) study, capturing data from 793 patients among 17 centers, the intraparenchymal hemorrhage rate was $2.4 \%$, while the aneurysm rupture rate was $0.6 \% .{ }^{32}$ In a recent meta-analysis of 3125 treated subjects, the calculated intraparenchymal hemorrhage and aneurysm rupture rates were $2.9 \%$ and $1.8 \%$, respectively. These complications are customarily thought to be higher in large or giant aneurysms. ${ }^{33}$ Calculated intraparenchymal hemorrhage and aneurysm rupture rates were $5.4 \%$ and $7.5 \%$, respectively, in giant aneurysms, and $2.1 \%$ and $1.3 \%$, respectively, in small and large aneurysms. The IntrePED study generated similar results, with intraparenchymal hemorrhage and aneurysm rupture rates of $5.8 \%$ and $5.8 \%$, respectively, in giant ICA aneurysms; $2.6 \%$ and $0.5 \%$, respectively, in large ICA aneurysms; and, $1.9 \%$ and $0 \%$, respectively, in small ICA aneurysms. We observed an overall hemorrhagic rate of $6.1 \%(5 / 82)$ in the Tubridge group, of which 2 hemorrhagic occurrences were thought to be the result of procedural injury. Excluding these 2 cases, 2/82 (2.4\%) manifested as aneurysm rupture. We did not encounter intraparenchymal hemorrhage, but we observed 1 (1.2\%) lateral Sylvian fissure SAH, unrelated to aneurysm rupture. The results of our study are very comparable with those of the above-described studies.

The prospect of ischemic stroke presents additional concerns. A complication rate of $9.68 \%$ in the Enterprise control group was consistent with that reported previously by Chalouhi et $\mathrm{al}^{29}$ as well as our own single-center experience, in the range of 7.58\%$11.4 \% .{ }^{34}$ Overall, ischemic rates after FD implantation vary among multiple publications, with a rate of $0 \%-10.3 \%$. Two recent meta-analyses indicated ischemic rates ranging from $5.5 \%$ to $7.5 \% .{ }^{33,35}$ However, the occurrence was increased in large or giant aneurysms due to intra-aneurysmal thrombosis or a prolonged procedure time, with a rate ranging from $5.2 \%$ to $13.5 \% .{ }^{32}$ Ye et $\mathrm{al}^{33}$ indicated an ischemic rate of $9.5 \%$ for giant aneurysms. Among Tubridge subjects of the PARAT trial, ischemic complications occurred in $8(9.76 \%)$ subjects. Although the PARAT trial rates are comparable with reported thromboembolic rates in published studies, a 9\%-10\% incidence of ischemic complications should be anticipated when using FDs for large or giant intracranial aneurysms. In the FIAT study, 12/75 subjects (16.0\%; 95\% CI, $8.9 \%-26.7 \%)$ treated by flow diversion were dead $(n=8)$ or dependent $(n=4)$ at $\geq 3$ months postimplantation. ${ }^{8}$ These results differ from those in our previous experience with FDs. We believe this discrepancy may reflect "real world" early experience with flow diverters for large or giant aneurysms in multiple centers with adjudication by an external imaging Core Laboratory. Similar reports of low procedure-related risks are seen commonly with single-center or retrospective studies, as best exemplified by the recent the Stenting vs. Aggressive Medical Management for Preventing Recurrent Stroke in Intracranial Stenosis (SAMMPRIS) trial. ${ }^{36}$

To better understand why these complications occurred, we undertook a detailed review of the PARAT trial complications. One possible factor is the role of the learning curve with flow diversion, and physicians' experience may still play a role in determining the relative safety of FD therapy. The prospect of a learning curve not only encourages a higher skill level in device handling and stent deployment but also leads to more appropriate subject selection. After gaining more experience with a new flow diverter in the treatment of large or giant aneurysms, physicians can avoid many technical failures, make more appropriate FDsize selections, better understand the necessity of appropriate postdeployment balloon dilation to avoid poor apposition or overdilation, and better identify the need for simultaneous coil insertion in the treatment of large or giant aneurysms. As an example, the PARAT trial experienced high rates of parent artery occlusions, and these contributed to 3 ischemic events in the Tubridge FD group. The possibility of malapposition may exist when the devices are underdeployed. In such instances, balloon dilation after initial FD deployment may protect against in-stent thrombosis and parent artery occlusion.

The concept of a PARAT trial learning curve was also supported by our multivariate analysis, which showed significantly lower complication rates in the primary investigational site, where the leading physicians already had gained considerable experience with the Tubridge FD in a previous single-center study. We believe that intensive training of inexperienced physicians on all flow diverters should be advocated before launching a study or introducing use in general neurointerventional practice. We believe that the role that learning curves can play in trials should be increasingly emphasized.

Several recent studies have suggested that antiplatelet regimens may play a role in the occurrence of hemorrhagic or thrombotic complications. ${ }^{37,38}$ In the PARAT trial, investigator management of antiplatelet therapy and subject compliance with prescribed study medications were not investigated.

We have identified some key limitations of the PARAT trial. The most obvious one is the high number of postconsent subject withdrawals $(n=41)$ in both arms, highlighting the delicate balance between physicians and subjects as to what treatment might 
be better suited for optimal outcomes in the treatment of highly challenging aneurysms. Seven prospective Tubridge subjects voiced their concerns about the potential adverse effects of flow-diverter use, while 15 subjects did not want to undergo stent-assisted coiling because they feared that the long-term durability was inferior to that anticipated with flow diverters. These subjects preferred to withdraw from the trial and wait to be treated with a commercially available flow diverter. The concept of new or apparently complicated technology can be intimidating for some patients.

Among the remaining subjects who departed from this trial prematurely, 1 dropped out after an incomplete subject consent, 6 had their index procedure terminated early after angiography examination indicated safety concerns, 1 was enrolled mistakenly, 1 violated the trial protocol and did not qualify for the index procedure, and 10 were terminated or withdrew from the trial for unknown reasons. Incomplete trial participation by the abovementioned subjects led to a need to expand the sample size and prevent unbalanced subject numbers and age. We compared the baseline data of subjects who were LTF with those from the full analysis set and determined that their removal had not affected the overall distribution of characteristics. Moreover, although subject age was not thought to affect outcomes, we used multivariable logistic regression, adjusted for subject age, to eliminate the effect of this imbalance. Second, the results of this study only represent the treatment of a specific subtype of aneurysms and a preliminary application of a novel FD. As discussed above, increasing familiarity with the Tubridge device is expected to lower the rate of clinical complications. Finally, although we included treatment of vertebral artery aneurysms in this trial, only 4 subjects with these aneurysms were enrolled. Thus, generalizations about the use of flow diverters in vertebral arteries should be made with caution. Clearly, additional vertebral artery aneurysm studies are warranted.

\section{CONCLUSIONS}

This trial showed that there was a significantly higher obliteration rate of aneurysms treated with the Tubridge FD versus conventional Enterprise SAC treatment in a selected group of patients with large or giant intracranial aneurysms. However, this higher obliteration rate comes at a cost of nonsignificant higher complication rates. For inexperienced operators who are either entering flow-diverter clinical trials or preparing for real-world clinical use, the importance of a learning curve should be emphasized.

\section{ACKNOWLEDGMENTS}

We thank Professors Ming-hua Li, Qiu-ping Li, and Liang Shao for their contribution in evaluating the 6-month angiographic results; Professors Yin Jian-hua, Chen Yao, Dong Lin, and DongLei Song for data and safety monitoring; and Professors XiaoYan Yan, Chong Ya Dong, and Yao Chen for their statistical analyses. Professor Jia He gave some guidance when drafting and revising this manuscript. We thank them for their constructive guidance. We are grateful to all principal investigators and delegated physicians who enrolled participants in all participating centers. We also thank the participants and their caretakers and families who consented to participate in the PARAT trial.

\section{REFERENCES}

1. International Study of Unruptured Intracranial Aneurysms Investigators. Unruptured intracranial aneurysm: risk of rupture and risks of surgical intervention. $N$ Engl J Med 1998;339:1725-33 CrossRef Medline

2. Barrow DL, Alleyne C. Natural history of giant intracranial aneurysms and indications for intervention. Clin Neurosurg 1995;42: 214-44 Medline

3. Darsaut TE, Darsaut NM, Chang SD, et al. Predictors of clinical and angiographic outcome after surgical or endovascular therapy of very large and giant intracranial aneurysms. Neurosurgery 2011;68: 903-15; discussion 915 CrossRef Medline

4. Gonzalez NR, Duckwiler G, Jahan R, et al. Challenges in the endovascular treatment of giant intracranial aneurysms. Neurosurgery 2008;62(6 Suppl 3):1324-35 Medline

5. Sluzewski M, Menovsky T, van Rooij WJ, et al. Coiling of very large or giant cerebral aneurysms: long-term clinical and serial angiographic results. AJNR Am J Neuroradiol 2003;24:257-62 Medline

6. Chalouhi N, Tjoumakaris S, Gonzalez LF, et al. Coiling of large and giant aneurysms: complications and long-term results of 334 cases. AJNR Am J Neuroradiol 2014;35:546-52 CrossRef Medline

7. Sadasivan C, Cesar L, Seong J, et al. Treatment of rabbit elastase-induced aneurysm models by flow diverters: development of quantifiable indexes of device performance using digital subtraction angiography. IEEE Trans Med Imaging 2009;28:1117-25 CrossRef Medline

8. Raymond J, Gentric JC, Darsaut TE, et al. Flow diversion in the treatment of aneurysms: a randomized care trial and registry. $J$ Neurosurg 2017;127:454-62 CrossRef Medline

9. Zhou Y, Yang PF, Fang YB, et al. A novel flow-diverting device (Tubridge) for the treatment of 28 large or giant intracranial aneurysms: a single-center experience. AJNR Am J Neuroradiol 2014;35:2326-33 CrossRef Medline

10. Kallmes DF, Brinjikji W, Boccardi E, et al. Aneurysm Study of Pipeline in an Observational Registry (ASPIRe). Interv Neurol 2016;5: 89-99 CrossRef Medline

11. Becske T, Kallmes DF, Saatci I, et al. Pipeline for uncoilable or failed aneurysms: results from a multicenter clinical trial. Radiology 2013; 267:858-68 CrossRef Medline

12. Byrne JV, Beltechi R, Yarnold JA, et al. Early experience in the treatment of intra-cranial aneurysms by endovascular flow diversion: a multicentre prospective study. PLoS One 2010;5. pii: e12492 CrossRef Medline

13. Nelson PK, Lylyk P, Szikora I, et al. The Pipeline embolization device for the intracranial treatment of aneurysms trial. AJNR Am J Neuroradiol 2011;32:34-40 CrossRef Medline

14. Kan P, Siddiqui AH, Veznedaroglu E, et al. Early postmarket results after treatment of intracranial aneurysms with the Pipeline embolization device: a U.S. multicenter experience. Neurosurgery 2012;71: 1080-87; discussion 87-88 CrossRef Medline

15. Wakhloo AK, Lylyk P, de Vries J, et al; Surpass Study Group. Surpass flow diverter in the treatment of intracranial aneurysms: a prospective multicenter study. AJNR Am J Neuroradiol 2015;36:98-107 CrossRef Medline

16. Yu SC, Kwok CK, Cheng PW, et al. Intracranial aneurysms: midterm outcome of Pipeline embolization device-a prospective study in 143 patients with 178 aneurysms. Radiology 2012;265:893-901 CrossRef Medline

17. Chiu AH, Cheung AK, Wenderoth JD, et al. Long-term follow-up results following elective treatment of unruptured intracranial aneurysms with the Pipeline embolization device. AJNR Am J Neuroradiol 2015;36:1728-34 CrossRef Medline

18. Becske T, Potts MB, Shapiro M, et al. Pipeline for uncoilable or failed aneurysms: 3-year follow-up results. J Neurosurg 2017;127:81-88 CrossRef Medline

19. ClinicalTrials.gov. Multicenter Randomized Trial on Selective Endovascular Aneurysm Occlusion With Coils Versus Parent Vessel Reconstruction Using the SILK Flow Diverter (MARCO POLO Post-

AJNR Am J Neuroradiol 39:807-16 May 2018 www.ajnr.org

815 
Market Clinical Investigation). https://clinicaltrials.gov/ct2/show/ NCT01084681. Accessed March 17, 2014

20. ClinicalTrials.gov. A Randomized Trial Comparing Flow Diversion and Best-standard Treatment: the FIAT Trial. https://www.clinicaltrials.gov/ ct2/show/NCT01349582. Accessed January 20, 2016

21. ClinicalTrials.gov. Multicenter Randomized Study for Medico-economic Evaluation of Embolization With Flow Diverter Stent in the Endovascular Treatment of Unruptured Saccular Wide-necked Intracranial Aneurysms. https://www.clinicaltrials.gov/ct2/show/NCT01811134. Accessed January 20, 2016

22. ClinicalTrials.gov. Complete Occlusion of Coilable Aneurysms. https://www.clinicaltrials.gov/ct2/show/NCT00777907. Accessed January 20, 2016

23. Turfe ZA, Brinjikji W, Murad MH, et al. Endovascular coiling versus parent artery occlusion for treatment of cavernous carotid aneurysms: a meta-analysis. J Neurointerv Surg 2015;7:250-55 CrossRef Medline

24. Zhou Y, Yang P, Zhang Y, et al. Posterior cerebral artery-posterior communicating artery (PCA-PComA) aneurysms: report of five cases and literature review. Neurol India 2012;60:228-30 CrossRef Medline

25. Arambepola PK, McEvoy SD, Bulsara KR. De novo aneurysm formation after carotid artery occlusion for cerebral aneurysms. Skull Base 2010;20:405-08 CrossRef Medline

26. Jafar JJ, Russell SM, Woo HH. Treatment of giant intracranial aneurysms with saphenous vein extracranial-to-intracranial bypass grafting: indications, operative technique, and results in 29 patients. Neurosurgery 2002;51:138-44; discussion 44-46 CrossRef Medline

27. Spetzler RF, Schuster H, Roski RA. Elective extracranial-intracranial arterial bypass in the treatment of inoperable giant aneurysms of the internal carotid artery. J Neurosurg 1980;53:22-27 CrossRef Medline

28. Morgan MK, Ferch RD, Little NS, et al. Bypass to the intracranial internal carotid artery. J Clin Neurosci 2002;9:418-24 CrossRef Medline

29. Chalouhi N, Tjoumakaris S, Starke RM, et al. Comparison of flow diversion and coiling in large unruptured intracranial saccular aneurysms. Stroke 2013;44:2150-54 CrossRef Medline

30. Brinjikji W, Murad MH, Lanzino G, et al. Endovascular treatment of intracranial aneurysms with flow diverters: a meta-analysis. Stroke 2013;44:442-47 CrossRef Medline

31. Fargen KM, Hoh BL, Welch BG, et al. Long-term results of Enterprise stent-assisted coiling of cerebral aneurysms. Neurosurgery 2012;71:239-44; discussion 244 CrossRef Medline

32. Kallmes DF, Hanel R, Lopes D, et al. International retrospective study of the Pipeline embolization device: a multicenter aneurysm treatment study. AJNR Am J Neuroradiol 2015;36:108-15 CrossRef Medline

33. Ye G, Zhang M, Deng L, et al. Meta-analysis of the efficiency and prognosis of intracranial aneurysm treated with flow diverter devices. J Mol Neurosci 2016;59:158-67 CrossRef Medline

34. Zhang Y, Zhou Y, Yang P, et al. Comparison of the flow diverter and stent-assisted coiling in large and giant aneurysms: safety and efficacy based on a propensity score-matched analysis. Eur Radiol 2016; 26:2369-77 CrossRef Medline

35. Zhou G, Su M, Yin YL, et al. Complications associated with the use of flow-diverting devices for cerebral aneurysms: a systematic review and meta-analysis. Neurosurg Focus 2017;42:E17 CrossRef Medline

36. Derdeyn CP, Chimowitz MI, Lynn MJ, et al; Stenting and Aggressive Medical Management for Preventing Recurrent Stroke in Intracranial Stenosis Trial Investigators. Aggressive medical treatment with or without stenting in high-risk patients with intracranial artery stenosis (SAMMPRIS): the final results of a randomised trial. Lancet 2014;383:333-41 CrossRef Medline

37. Delgado Almandoz JE, Crandall BM, Scholz JM, et al. Last-recorded $\mathrm{P} 2 \mathrm{Y} 12$ reaction units value is strongly associated with thromboembolic and hemorrhagic complications occurring up to 6 months after treatment in patients with cerebral aneurysms treated with the Pipeline embolization device. AJNR Am J Neuroradiol 2014;35: 128-35 CrossRef Medline

38. Skukalek SL, Winkler AM, Kang J, et al. Effect of antiplatelet therapy and platelet function testing on hemorrhagic and thrombotic complications in patients with cerebral aneurysms treated with the Pipeline embolization device: a review and meta-analysis. J Neurointerv Surg 2016;8:58-65 CrossRef Medline

39. McAuliffe $\mathrm{W}$, Wycoco V, Rice $\mathrm{H}$, et al. Immediate and midterm results following treatment of unruptured intracranial aneurysms with the pipeline embolization device. AJNR Am J Neuroradiol 2012; 33:164-70 CrossRef Medline 